\title{
The co-occurrence of autism and attention deficit hyperactivity disorder in children - what do we know?
}

\author{
Yael Leitner ${ }^{1,2 *}$ \\ ${ }^{1}$ Child Development Center, Dana-Dwek Children's Hospital, Tel Aviv Sourasky Medical Center, Tel Aviv, Israel \\ 2 Sackler School of Medicine, Tel Aviv University, Tel Aviv, Israel
}

\section{Edited by:}

Itai Berger, Hadassah-Hebrew University Medical Center, Israel

\section{Reviewed by:}

Adriana Di Martino, New York University Langone Medical Center, USA

Itai Berger, Hadassah-Hebrew University Medical Center, Israel Linda Sprenger, Philipps-University Marburg, Germany

\section{*Correspondence:}

Yael Leitner, Child Development Center, Dana-Dwek Children's

Hospital, Tel Aviv Sourasky Medical Center, Weitzman St. 6, Tel Aviv

6423906, Israel

e-mail: leitnery@t/vmc.gov.il
Symptoms of attention deficit hyperactivity disorder (ADHD) and autistic spectrum disorder (ASD) often co-occur. The DSM-IV had specified that an ASD diagnosis is an exclusion criterion for $A D H D$, thereby limiting research of this common clinical co-occurrence. As neurodevelopmental disorders, both ASD and ADHD share some phenotypic similarities, but are characterized by distinct diagnostic criteria. The present review will examine the frequency and implications of this clinical co-occurrence in children, with an emphasis on the available data regarding pre-school age. The review will highlight possible etiologies explaining it, and suggest future research directions necessary to enhance our understanding of both etiology and therapeutic interventions, in light of the new DSM-V criteria, allowing for a dual diagnosis.

Keywords: autistic spectrum disorders, attention deficit hyperactivity disorder, diagnostic and statistical manual, co-morbidity, co-occurrence

\section{INTRODUCTION}

In the last decade, studies have reported increased prevalence of both attention deficit hyperactivity disorder (ADHD) and autistic spectrum disorders (ASD). While ADHD is defined by impaired functioning in the areas of attention, hyperactivity, and impulsivity, whereas ASD is characterized by core social dysfunction and restrictive-repetitive behaviors, studies show that between 30 and $50 \%$ of individuals with ASD manifest ADHD symptoms (particularly at pre-school age), and similarly, estimates suggest two-thirds of individuals with ADHD show features of ASD (Davis and Kollins, 2012). Recent findings from the Autism Treatment Network (ATN) database suggest that co-occurrence of ADHD and ASD is associated with a lower quality of life and poorer adaptive functioning than in any one of these conditions (Vora and Sikora, 2011). Both disorders often include difficulties in attention, communication with peers, impulsivity, and various degrees of restlessness or hyperactivity. Both are more common in boys than in girls, and present, at least partially, at pre-school age. Both disorders have a known genetic pre-disposition, with comorbidity within the same individual and across family members, and both syndromes cause significant behavioral, academic, emotional, and adaptive problems in school, at home, and elsewhere. (Rao and Landa, 2013).

Evidence for common neurobiological substrates has been found through similarities in neuropsychological profiles in individuals with both disorders (Gargaro et al., 2011; Rommelse et al., 2011). It has been shown that children with ADHD have pragmatic language difficulties similar to children in the ASD spectrum (Bishop and Baird, 2001). Further neuropsychological similarities are suggested by a study of emotional recognition and theory of mind, which showed that children with ADHD could not be distinguished from those with ASD (Buitelaar et al., 1999). A study on social perspective taking showed children with ADHD used lower levels of social perspective taking coordination in their definition of problems, identification of feelings, and evaluation of outcomes than children without ADHD, and these differences persisted after the role of language abilities, intelligence, and oppositional and conduct problems were taken into account (Marton et al., 2009).

Despite the growing body of research pointing at the frequent co-occurrence of these two disorders, the previous DSM-IV-TR has not allowed a dual diagnosis. The DSM-V, in its revised ADHD diagnostic criteria, recognizes the frequency of this co-occurrence and allows, for the first time, a co-morbid diagnosis of ADHD with autism spectrum disorder. This new attitude will not only allow for more efficient clinical management of these children, but will also clear the way for a more precise scientific understanding of the overlap of these two disorders. While most research to date has documented developmental trajectories for ADHD and ASD separately, little is known regarding their co-occurrence, particularly at young pre-school age. PUBMED was searched using the definition "co-occurrence of ASD and ADHD in pre-school children." This led to only 35 studies, and therefore search was broadened to include the more general definition of the "co-occurrence of ASD (or social-communication difficulties) and ADHD in children." More than 150 articles were eventually reviewed.

\section{ADHD (SYMPTOMS) IN CHILDREN WITH ASD}

A significant percentage of children with ASD seeking services at clinical centers present with comorbid symptoms of ADHD, with rates ranging between 37\% (Gadow et al., 2006) and 85\% (Lee and Ousley, 2006) across studies conducted in the United States 
and Europe (Rao and Landa, 2013). ADHD was the third most common disorder identified in a community sample of 5-17 years old children (Leyfer et al., 2006), with $31 \%$ of the sample meeting full ADHD criteria and another $24 \%$ with subsyndromal ADHD symptoms. This is lower than reported rates of ASD and ADHD in clinic samples (Rao and Landa, 2013). Very few studies have looked at the epidemiology of co-existing disorders in pre-school age children diagnosed with ASD. Two year-old twins $(n=312)$ from the Boston University Twin project were studied by Ronald et al. (2010) for autistic-like traits and ADHD behaviors using Child Behavior Checklist (CBCL) answered by their parents. Controlling for cognitive abilities and socioeconomic status, autistic like traits correlated positively with ADHD behaviors $(r=0.23-$ 0.26 ), a lower correlation than described for older children. In a recent survey by Carlsson et al. (2013), 198 pre-school Swedish children (age 4.5-6.5 years) with ASD who treated in a habilitation center, were assessed for such disorders. They found language problems in $78 \%$, intellectual disability in $49 \%$, below average motor function in $37 \%$, and severe hyperactivity in $33 \%$.

Lecavalier et al. (2009) has lately shown the validity of DSMIV syndromes in a group of 229 pre-schoolers with ASD, using confirmatory factor analysis (CFA). Despite very high factor loading with the general ADHD combined factor, several items had non-significant loadings with the specific ADHD hyperactiveimpulsive factor. In other words, the verbal items from the hyperactive-impulsive sub scale were negatively correlated with the other motor items suggesting hyperactivity-impulsivity has two components: physical and verbal. The authors comment that this is a good example of how the clinical features of ASD might alter the clinical presentation of a co-occurring DSM-IV defined (pre-school) ADHD. In a study by Sikora (Sikora et al., 2012), as part of the Autism Speaks ATN, data was collected from 14 sites in the US and Canada. Participants between the age of 2 and 17.9 years were included if they met DSM-IV and/or Autism Diagnostic Intervention Schedule (ADOS) diagnostic criteria for ASD, if they were cared for at an established ATN site, if parents was fluent in English, and it was the language spoken with the child at least $75 \%$ of the time. Parents were asked to complete the CBCL and the pediatric quality of life inventory (PedsQL) and were also interviewed to complete the Vineland Adaptive Behavior Scales (VABS-II). Cognitive scores were used as covariates. Of the whole study group, 1737 participants were $2-5$ years old. Of these, $40 \%$ had elevated $\mathrm{T}$ score in 1 of the 2 ADHDrelated scales, and $18.8 \%$ had both $\mathrm{ADHD}$-related scales $\mathrm{T}$ scores (>70) elevated. The ASD + ADHD group had lower scores on both the VABS-II and the PedsQL. The authors conclude that over one third of children with ASD have some comorbid ADHD symptoms, and their presence is related to greater problems in adaptive skills and poorer overall quality of life, and suggest primary care providers should screen for symptoms of ADHD in their patients with ASD, and consider these symptoms when developing a care plan.

\section{ASD SYMPTOMS (SOCIAL-COMMUNICATION DIFFICULTIES) IN CHILDREN WITH ADHD}

Social problems are not part of the core diagnostic criteria for ADHD, but children with ADHD experience significant social difficulties (Cantwell, 1996; Friedman et al., 2003). ADHD children are more often rejected by their peers, and have fewer friends (Hoza et al., 2005; Mikami, 2010). In many cases, these difficulties are viewed as a direct result of the ADHD core symptoms. Inattentive behaviors may lead a child to miss social cues, impulsiveness may result in upsetting peers, and hyperactivity hinders participation in organized activities and leads to avoidance of peers. It is estimated that approximately $50-60 \%$ of ADHD children experience rejection by their peers (Barkley et al., 1990). In fact, many ADHD children are disliked within minutes of the initial social interaction (Pelham et al., 1985) and then denied further opportunities to practice social skills, which lead to further rejection (Milich et al., 1982). Specific play behaviors have been linked with rejection of ADHD children and include being bossy, intrusive, inflexible, controlling, annoying, explosive, argumentative, easily frustrated, inattentive during organized sports/games, and violating the rules of the game (Pelham et al., 1985; Whalen et al., 1985; Klassen et al., 2004; Young et al., 2005). Social functioning by ADHD subtype varies somewhat according to rater (e.g., teachers, parents, and peers); however, the general consensus is that all ADHD subtypes are at risk for peer rejection (Carlson and Mann, 2000; Hodgens et al., 2000). The presence of co-morbid psychiatric disorders tends to exacerbate social impairments in children with ADHD (Greene et al., 1996; Antshel and Remer, 2003). This is significant when considering that over $2 / 3$ of individuals with ADHD have a co-morbid psychiatric disorder (Cantwell, 1996) with rates reported to be $15-75 \%$ with mood disorder, $25 \%$ with anxiety, and 30-50\% with conduct disorder (CD). Others (Biederman et al., 1991; Eiraldi et al., 2000) have found that anxiety and depression together accounted for $30 \%$ of the variance in social impairment in ADHD. Children with both ADHD and a learning disability have also been found to have greater peer relations difficulties than children with only a learning disability (Flicek, 1992).

This profile of social difficulties differs, however, from that observed in ASD, in which impairment in the basic understanding of social realm is central, such as a lack of emotional reciprocity and engagement with others, and the low interest or enjoyment in social interaction. Cantwell (1996) described a type of social difficulty in ADHD by a "lack of savoir faire," and estimated that this social naivety may affect some $20 \%$ of ADHD children and adolescents. Recent research suggests that many individuals with ADHD may experience social impairments that are more consistent with those observed in ASD. In children with a primary diagnosis of $\mathrm{ADHD}$, the level of autistic symptomatology corresponded to the severity of ADHD subtype; children with the combined type of ADHD-demonstrated the most autistic symptoms (Reiersen et al., 2007). In a study recently published by Kotte et al. (2013), a positive autistic traits (AT) profile operationalized from the CBCL, was significantly overrepresented among ADHD children vs. controls (18 vs. $0.87 \% ; P<0.001$ ).

Recent research suggests many individuals with ADHD may experience social impairments consistent with those observed in ASD. Santosh and Mijovic (2004) characterized the social impairments in children with ADHD as associated with either relationship difficulty (conduct and affective problems), or with social-communication difficulty. Children with the latter were 
more likely to exhibit repetitive behaviors, speech and language impairment, and developmental problems similar to ASD. Other investigators have described deficient empathy and facial affect recognition in children with ADHD (Sinzig et al., 2008; Uekermann et al., 2010). Other studies have also pointed at the increased rate of autistic symptoms in samples of children with ADHD. Grzadzinski et al. (2011) confirmed the presence of a sub group of children with ADHD and elevated ratings of core ASD traits not accounted for by ADHD or behavioral symptoms. The ADHD group with AT revealed greater ODD behaviors than those with ADHD-only. Most of the studies conducted in middle or late childhood have shown that a substantial proportion of children with ADHD show significant autistic symptoms (Santosh and Mijovic, 2004; Holtmann et al., 2007; Nijmeijer et al., 2008). Very few studies have looked at pre-school children with ADHD, in an effort to identify early "comorbid" ASD symptoms, perhaps because an ASD diagnosis is usually made at pre-school age, while a primary ADHD diagnosis is often delayed to late pre-school or early school age.

\section{IMPACT OF COMORBID ADHD AND ASD}

Diagnostic constraints have limited research on co-occurring ADHD and ASD, because many studies, in accordance with the DSM-IV, have excluded individuals with other psychiatric or developmental difficulties (Davis and Kollins, 2012). There is preliminary evidence that when ADHD is comorbid with ASD, the risk for increased severity of psychosocial problems increases (Gadow et al., 2004; Yerys et al., 2009). Research comparing individuals with both diagnoses to individuals with a single diagnosis suggest that co-occurring symptoms are associated with greater impairment than a single diagnosis. By both parent and teacher reports children with ADHD and ASD (Rao and Landa, 2013) experience more difficulty in daily life. Furthermore, these co-occurring conditions may be less responsive to standard treatments for either disorder. Children 4-8 years old with ASD, whose parents report significant symptoms of ADHD, show lower cognitive functioning, more severe social impairment, and greater delays in adaptive functioning than children with ASD-only.

Sikora et al. as part of the activities of the ATN (Sikora et al., 2012) collected data on children with ASD across 14 sites in the US and Canada. Children were between ages 2 and 17.9 years. They were divided into groups based on whether their parents rated them as having clinically significant scores on ADHD problems subscales from the CBCL. $56.6 \%$ of the sample was young children between 2 and 5 years. Analysis revealed that those with ASD+ADHD symptoms had lower scores in all the areas measured. Psychosocial health summary, school functioning, physical functioning, emotional, and social functioning scores were all lower than those of the children with ASD alone $(P<0.0001)$. In another study by Gadow et al. (2004), PDD and non-PDD clinic groups showed equally severe $\mathrm{ADHD}$ and oppositional defiant disorder symptoms. As measured by parent and teacher referenced rating scale (ECI-4), Mulligan et al. (2009) compared autism symptoms in $821 \mathrm{ADHD}$ probands, 1050 siblings, and 149 controls by using the Social-Communication Questionnaire (SCQ). Latent class analysis yielded five classes; class 1 (31\%) had very few autism symptoms and low comorbidity; classes 2-4 were intermediate; class 5 (7\%) had high autism symptoms and comorbidity. The cluster with the highest mean SCQ score (class 5) had the highest prevalence of co-morbid oppositional defiant disorder, CD, language disorder, and motor disorder. Other evidence supports more ODD symptoms in children with both disorders when using teacher rating rather than parent ratings (Guttmann-Steinmetz et al., 2009). These findings, however, describe mostly school age children, while there is a lack of psychiatric comorbidity studies in younger children with both conditions.

Several investigators have found (Sinzig et al., 2008; Rao and Landa, 2013) a higher percentage of children with both ASD+ADHD were classified as having significant cognitive delays than children with ASD-only (61 vs. 25\%). Kotte et al. (2013) had reported ADHD children with high score on the AT profile of the CBCL, were significantly more impaired than control subjects in psychopathology, interpersonal, school, family, and cognitive domains. Yerys et al. (2009) reported on elevated rates of externalizing problem behaviors as well as greater impairment in executive functioning in children with comorbid ASD and ADHD, compared with children with ASD-only. Children with high functioning autism (HFA) and attention problems scored significantly below the children with HFA only, on the verbal memory and delayed recall measures, supporting the proposition that children with both disorders differ not only on a clinical level but also on a neurocognitive level (Andersen et al., 2013).

Only one study has looked at the relationship between the two disorders as they develop over time. St Pourcain et al. (2011) have followed 5,383 singletons (the Avon Longitudinal Study of Parents and Children-ALSPAC) ages 4-17 years and assessed multiple measures of hyperactive-inattentive traits and autistic social-communication impairment at multiple time points. Autistic symptoms were more stable than those of ADHD behaviors, which showed greater variability. Trajectories for both traits were strongly, but not reciprocally interlinked, such that the majority of children with a persistent hyperactive-inattentive symptomatology also showed persistent social-communication deficits, but not vice versa. Shared predictors, especially for trajectories of persistent impairment were maternal smoking during the first trimester, which included familial effects and a teenage pregnancy. The authors conclude that patterns of association between ASD and ADHD symptoms change over time, and propose to remove exclusivity criteria for these diagnoses in the DSM-V, as has already been done.

\section{POSSIBLE ETIOLOGIES FOR THE CO-OCCURRENCE OF ASD AND ADHD}

Due to the previous DSM-IV diagnostic constraints, research concerning the possible etiologies of the co-occurrence of ADHD and ASD is scarce. The central focus of available research is in the fields of neuropsychology, genetics, and neuroimaging.

Although there are some important differences between the two disorders, as mentioned in the introduction, ASD and ADHD share many similar impairments in developmental and cognitive domains. Both are more common in males, have a strong comorbidity with intellectual disability, and are also associated with other specific learning and developmental difficulties, notably language, reading, and motor problems. Executive functions (EF) deficits 
are common in both disorders, together with response inhibition deficit. EF measures hardly discriminated between ADHD and HFA, but compared to children with ADHD, the HFA group showed more difficulty with cognitive flexibility and planning (Cooper et al., 2014). Children with ADHD have pragmatic language difficulties similar to children in the ASD spectrum (Bishop and Baird, 2001). Further neuropsychological similarities are suggested by a study of emotional recognition and theory of mind which showed that children with ADHD could not be distinguished from those with ASD (Buitelaar et al., 1999).

Rommelse et al. (2010) suggest a variety of hypotheses to explain co-occurrence, but the two most likely explanations may be that the two are independent disorders occurring together by association with a third independent factor, or alternatively they share a common underlying etiology. The authors believe the latter is the most likely model and that both disorders share a common genetic basis. Their view is supported by several family, twin, and molecular genetic studies. Both family (Holtmann et al., 2007; Guttmann-Steinmetz et al., 2009) and twin studies (Reiersen et al., 2007; Ronald et al., 2010) provide support for the hypothesis that ADHD and ASD originate from partly similar familial/genetic factors. About $50-72 \%$ of the contributing genetic factors in both disorders show overlap. These shared genetic and neurobiological underpinnings form an explanation why both disorders occur so frequently within the same patient and family.

Two related family studies originating from the IMAGE (Holtmann et al., 2007; Guttmann-Steinmetz et al., 2009) cohort have examined the rates and severity of ASD in probands with ADHD and their siblings. Mulligan et al. (2009) measured autism symptoms using the SCQ and compared 821 ADHD probands, 1050 siblings, and 149 controls. Affected and unaffected male (but not female) siblings had higher ASQ scores than controls. The phenotypic correlation between ASD and ADHD was slightly higher for males (0.63) than for females (0.49). Using a modified method of the deFries-Fulker analysis, the authors conclude that $56 \%$ of the cross-correlation $(=0.18)$, could be explained by shared genetic influences on ADHD and ASD. In another study by Nijmeijer et al. (2008), using the same cohort, 256 sibling pairs and 147 controls were studied, using the Children Social Behavior Questionnaire (CSBQ). This instrument measures less severe variants of ASD. Similar to Mulligan's study, sibling correlations for ASD were significant, but contrary to the earlier study, correlations were higher in female probands (0.44), than male probands (0.23). Also, siblings correlations for ASD were higher in older than younger children, indicating an increased genetic influence on ASD within ADHD families over time. In this study, cross correlations were not significant, suggesting independent familial factors give rise to ADHD and ASD.

In a twin study, mentioned earlier (Reiersen et al., 2007), AT were examined in 8 years old ADHD twins $(N=6771)$, using parent and teacher questionnaires. Phenotypic correlations were 0.54 for parent and 0.51 for teacher data. The authors concluded that genetic correlations between ASD and ADHD symptoms were all $>0.50$. Similar results were reported in a study of young adult twins $(N=674)$, using a self reported measures of ADHD and ASD. A bivariate model indicated that the genetic correlation between ADHD and ASD was 0.72 . Models with additive genetic effects and unique environmental effects fitted the data best, with no evidence for sex differences. While the limitation of most of these studies is the use of rating scales rather than direct clinical measures to evaluate either ADHD or ASD, together they do point at a genetic correlation between the two diagnoses.

Linkage studies and Genome Wide Association Studies (GWAS) have specifically addressed this co-occurrence, pinpointing to some promising pleiotropic genes, loci, and single nucleotide polymorphisms (SNPs). Their authors comment that there is an urgent need for better designed and powered studies to tackle this complex issue. A recent study (Cross-Disorder Group of the Psychiatric Genomics Consortium, 2013) using GWAS data from the Psychiatric Genomic Consortium (PGC), for cases and controls in five psychiatric disorders including ADHD and ASD, did not find any significant genetic correlation between ASD and ADHD, using common SNP's. Analyses revealed that there were modest shared genetic influences between ADHD and AT as well as some common environmental influences explaining their co-variability. The contribution of assortative mating and parent-of-origin effects to the co-occurrence of ASD and ADHD has been investigated by van Steijn et al. (2012) in 121 families with at least one child diagnosed with ASD, and one or more biological sibling. All children and parents were carefully screened for the presence of ASD and ADHD. The authors concluded that cross-assortative mating for ASD or ADHD does not explain the frequent co-occurrence of these disorders within families. They did show, however, that parental ADHD is predictive of offspring' ASD but not vice versa, hinting that risk factors underlying ASD may overlap to a larger degree with risk factors underlying ADHD than vice versa.

In autism, as opposed to ADHD, anatomical studies found larger total brain and white matter volumes in most cortical brain regions and in the cerebellum, caudate, and globus pallidum (Piven et al., 1996). A shared anatomical dysmorphology between the two disorders appears to be a smaller corpus callosum. In functional neuroimaging, the most consistent findings has been that of reduced frontal and parietal activation across a wide range of tasks in ASD (Baron-Cohen et al., 1999), which may not be different for ADHD. Geurts et al. (2013) tested whether there is a relationship between gray matter (GM) volume and autism and ADHD symptom severity by using structural MRI. They found that selfreports on symptom severity of both disorders correlated with GM volume in the left inferior frontal gyrus, but each disorder symptom severity was correlated separately with different cortical areas. They conclude it seems to be an oversimplification to typify psychiatric disorders solely as extremes of brain structure abnormalities. Di Martino et al. (2013) used voxel wise network centrality, functional connectivity metrics indexing local [degree centrality (DC)] and global (eigenvector centrality) functional relationships across the entire brain connectome, in resting state functional magnetic resonance imaging data from 56 children with ASD, 45 children with $\mathrm{ADHD}$, and 50 typically developing children. In both clinical groups, cortical and sub cortical areas exhibited centrality abnormalities, some common to both disorders (in the precuneus), but others were disorder-specific. Secondary analyses differentiating children with ASD into those with or without ADHD-like comorbidity $[\operatorname{ASD}(+)$ and $\operatorname{ASD}(-)$, respectively] revealed that the $\mathrm{ASD}(+)$ group shared ADHD-specific abnormalities in basal 
ganglia. By contrast, centrality increases in temporolimbic areas characterized children with ASD regardless of ADHD-like comorbidity. The authors conclude that their work provides evidence for both shared and distinct underlying mechanisms at the large-scale network level. These and other neuroimaging studies in the future might lead to better understanding of the neuro-circuitry involved in the co-occurrence of ASD and ADHD.

\section{TREATMENT OF CO-OCCURRING ADHD AND ASD}

Much evidence supports the use of medications to treat symptoms of ADHD in typically developing school age children (Subcommittee on Attention Deficit/Hyperactivity Disorder et al., 2011). ADHD medications have shown their efficacy in pre-school children (PATS study), but with less efficacy and more side effects than in school age children (Riddle et al., 2013). For ASD, medications target comorbid behavioral symptoms like irritability, hyperactivity, and aggression, rather than the core social and communication deficits. Several medications have demonstrated the potential to treat repetitive/stereotyped behaviors, but efficacy data has not been strong. Only two medications, to date, have been formally approved for use in ASD, and they both target irritability; Risperidone and Aripiprazole (Abilify) (Owen et al., 2009; Canitano and Scandurra, 2011; Williams et al., 2013). Despite limited research on the pharmacological treatment in ASD, there is a significant increase in the use of psychoactive medications in this group in recent years, in part due to an increase in the use in ADHD medications in ASD children with ADHD symptoms. Frazier et al. (2011) have recently used data on psychotropic medication from the first wave of the National Longitudinal Transition Study 2, a nationally representative study of adolescents ages 13-17 in special education, and have shown that youths with ASD+ADHD had the highest rates of use (58.2\%), followed by youths with ADHD-only (49.0\%) and youths with ASD-only (34.3\%). Youths with ASD, both ASD-only and ASD + ADHD, used medications across a variety of medication classes, whereas stimulants were dominant among youths with ADHD-only.

\section{STIMULANT MEDICATIONS}

Methylphenidate and atomoxetine (Frazier et al., 2011) are both typically used to treat ADHD and are also effective in ASD. Santosh et al. (2006) conducted a retrospective and an open-label prospective trial to compare response to stimulants (methylphenidate or dextroamphetamine) between children with ASD and ADHD and children with ADHD alone, and found no significant differences in treatment response or side-effect profiles between groups. However, other studies suggest that response rates of methylphenidate may differ in ASD as compared to what is reported in typically developing children with ADHD alone. The National Institute of Mental Health Collaborative Multisite Multimodal Treatment Study of Children with ADHD (MTA) (MTA Cooperative Group, 2004) reported response rates of $70-80 \%$ as compared to the $49 \%$ reported in the Research Units of Pediatric Psychopharmacology (RUPP) Autism Network trial of methylphenidate (Arnold et al., 2012). In terms of tolerability, $18 \%$ of subjects in the RUPP trial withdrew, yet discontinuation rates were quite low in the MTA study (1.4\%). While methylphenidate may improve irritability in ADHD without ASD, it appears to worsen irritability in some patients with ASD.

Reduction in ADHD symptoms has been the primary outcome measure of most studies of co-occurring ADHD and ASD. A secondary analysis of the RUPP evaluated the effects of methylphenidate on social-communication skills and selfregulation in 33 children with ASD. Weekly dedicated observations over a 4-week period, indicated that methylphenidate use was associated with several positive social outcomes; including improved initiation of joint attention, improved response to bids for joint attention, and better affective and self-regulation.

\section{NON-STIMULANT MEDICATIONS}

In the only controlled study of atomoxetine (Jahromi et al., 2009), results were significantly better than placebo, but the sample size was small and only 7 of 16 children $(43 \%)$ were considered responders. Overall, both methylphenidate and atomoxetine appear to effectively treat ADHD-related symptoms in ASD (MTA Cooperative Group, 2004; Arnold et al., 2006, 2012; Posey et al., 2006; Santosh et al., 2006; Jahromi et al., 2009; Murray, 2010), but atomoxetine demonstrated better tolerability than stimulants in individuals with co-occurring ADHD and ASD. Response rates may, however, be lower in ASD plus ADHD, than in ADHD alone, and symptoms of inattention may be less likely to respond than symptoms of hyperactivity and impulsivity. Atomoxetine effectiveness may vary as a function of level of impairment, measured by cognitive ability or by ASD symptom severity (Arnold et al., 2006; Posey et al., 2006). Finally, treatment success may be limited by tolerability. Many studies have demonstrated efficacy for antipsychotics, and since the RUPP trial with risperidone this medication in particular has consistently shown benefit for hyperactivity in ASD (Murray, 2010; Matson et al., 2011; Sharma and Shaw, 2012). In 2006, The United States Food and Drug Administration approved risperidone for the treatment of irritability in ASD in children and adolescents 5-16 years of age. However, significant concerns about tolerability remain and suggest that benefits of this medication must be carefully weighed against the risks. Evidence from controlled studies of alpha-2 agonists for ADHD-related symptoms in ASD is inconsistent and response rates are relatively low. Open-label studies of guanfacine (Intuniv) appear promising but additional controlled studies are needed. A retrospective analysis (Posey et al., 2004) of 80 patients indicated reduction in hyperactivity and inattention among children with ASD and higher cognitive functioning. Alpha-2 agonists may be a reasonable alternative or augmentation strategy and have the advantage of being relatively benign. Amantadine may be considered in the treatment of ASD but its use for ADHD-related symptoms is not yet supported by research (Hosenbocus and Chahal, 2013). Other medications like melatonin, antioxidants, acetylcholinesterase inhibitors, and naltrexone, are currently considered off-label for ASD, but further randomized controlled trials are necessary (Rossignol, 2009).

For all medication categories except MPH, no studies are available on pre-school children. 


\section{PSYCHOSOCIAL INTERVENTIONS}

An up to date literature survey on psychosocial interventions in children with both ADHD and ASD has not revealed any results. In a comprehensive review on the treatment of these cooccurring conditions Davis and Kollins (2012) mention that there are similarities across approaches to treat both disorders. In both, treatment uses conditioning procedures, which have evolved in time to draw on a social learning theory (Brookman-Frazee et al., 2006). Whereas both ADHD and ASD include behaviorally oriented parental intervention, the role of the family is conceptualized in a different way; for ADHD "parent training" involves teaching parents to manage the behaviors of their children, in ASD "parent education" places more emphasis on individualized treatments that provide parents with tools to promote their child's (social) skill development. Davis and Kollins suggest that bridging between these two strategies might benefit those with comorbid disorders.

While many studies have shown the importance of combining medication and psychosocial interventions (mostly parental education) for children with ADHD, there are only a few studies on the combined medication and behavioral approach in ASD children. Aman et al. (2009) primarily targeted frequent tantrums, self-injury, and aggression in a trial of risperidone treatment and parent training, but the combined effects on hyperactivity were also examined. Results indicated that children who received the combined treatments had lower rates of aggression and greater reduction in hyperactivity (requiring lower risperidone dose), as compared with children who received medication only.

Studies on psychosocial interventions in pre-school children with co-occurring ASD and ADHD are lacking.

\section{SUMMARY AND FUTURE DIRECTIONS}

Research specifically focusing on co-occurring ADHD and ASD has only recently evolved, being previously limited by the DSM-IV exclusion criteria. What we have so far learned is that both disorders frequently co-occur, and when they do, they cause greater morbidity, and create a more complicated clinical challenge. The new DSM-V, allowing for a dual diagnosis, will hopefully facilitate research, by eliminating the exclusion of many patients and allowing the study of broader phenotypes. Most recent research focuses on etiology and clinical presentations, with less direct work on treatment and early intervention protocols. Very few studies have looked at pre-school children presenting with both conditions, on the impact of early intervention in this age group and its effect on developmental trajectory. Furthermore, many of the above quoted studies have used parent and/or teacher rating scales to assess clinical profiles, rather than direct clinical diagnoses of the two co-existing disorders. In the future, as a dual diagnosis is "officially" possible, studies using direct clinical assessment of both comorbid diagnoses are essential.

Future research should tackle two major hypotheses regarding the frequent co-occurrence of ASD and ADHD. The first is that $\mathrm{ADHD}$ and Autism are distinct, yet overlapping disorders which may share some common etiology, probably genetic. The second hypothesis is that the co-occurrence of autistic symptoms and ADHD "stands alone" as a distinct clinical disorder, with a distinct etiology, and a different developmental trajectory. These two hypotheses should be examined by studying the developmental trajectories in co-occurring ASD and ADHD, by defining common co-morbidity profiles in both, and by understanding differences and similarities in social perception, motor functions and language, cognition, and EF in each disorder and in the co-occurring "phenotype." Defining early "endophenotypes" as suggested by Rommelse et al. (e.g., heritable vulnerability traits that form a link between genes and observable symptoms, e.g., neuroimaging, neuropsychological functions) of both disorders, should serve to increase the chance of identifying genetic markers for each one and for both together (Cross-Disorder Group of the Psychiatric Genomics Consortium, 2013).

In 2010, Gillberg et al. have coined the ESSENCE concept (Gillberg, 2010) an acronym for early symptomatic syndromes eliciting neurodevelopmental clinical examinations, pointing to the fact that major problems in at least one developmental domain before age 5 years often signals major problems in the same or overlapping domains years later. They suggest "There is no time to wait," implicating intervention should be the main goal, and not necessarily the categorical diagnosis. They go on to suggest intervention should be broad, addressing the multiple aspects of developmental disorders at this young age. Future studies should focus on early identification and intervention strategies in this specific "co-morbid" group, with an emphasis on pre-school children, using prospective design, even before pathophysiology is fully understood.

\section{REFERENCES}

Aman, M. G., McDougle, C. J., Scahill, L., Handen, B., Arnold, L. E., Johnson, C., et al. (2009). Medication and parent training in children with pervasive developmental disorders and serious behavior problems: results from a randomized clinical trial. J. Am. Acad. Child Adolesc. Psychiatry 48, 1143-1154. doi:10.1097/CHI.0b013e3181bfd669

Andersen, P. N., Hovik, K. T., Skogli, E. W., Egeland, J., and Oie, M. (2013). Symptoms of ADHD in children with high-functioning autism are related to impaired verbal working memory and verbal delayed recall. PLOS ONE 8:e64842. doi:10.1371/journal.pone.0064842

Antshel, K. M., and Remer, R. (2003). Social skills training in children with attention deficit hyperactivity disorder: a randomized-controlled clinical trial. J. Clin. Child Adolesc. Psychol. 32, 153-165. doi:10.1207/15374420360533149

Arnold, L. E., Aman, M. G., Cook, A. M., Witwer, A. N., Hall, K. L., Thompson, S., et al. (2006). Atomoxetine for hyperactivity in autism spectrum disorders: placebo-controlled crossover pilot trial. J. Am. Acad. Child Adolesc. Psychiatry 45, 1196-1205. doi:10.1097/01.chi.0000231976.28719.2a

Arnold, L. E., Aman, M. G., Li, X., Butter, E., Humphries, K., Scahill, L., et al. (2012). Research Units of Pediatric Psychopharmacology (RUPP) Autism Network randomized clinical trial of parent training and medication: one-year follow-up. J. Am. Acad. Child Adolesc. Psychiatry 51, 1173-1184. doi:10.1016/ j.jaac.2012.08.028

Barkley, R. A., DuPaul, G. J., and McMurray, M. B. (1990). Comprehensive evaluation of attention deficit disorder with and without hyperactivity as defined by research criteria. J. Consult. Clin. Psychol. 58, 775-789. doi:10.1037/0022-006X.58.6.775

Baron-Cohen, S., Ring, H. A., Wheelwright, S., Bullmore, E. T., Brammer, M. J., Simmons, A., et al. (1999). Social intelligence in the normal and autistic brain: an fMRI study. Eur. J. Neurosci. 11, 1891-1898. doi:10.1046/j.1460-9568.1999. 00621.x

Biederman, J., Newcorn, J., and Sprich, S. (1991). Comorbidity of attention deficit hyperactivity disorder with conduct, depressive, anxiety, and other disorders. Am. J. Psychiatry 148, 564-577.

Bishop, D. V., and Baird, G. (2001). Parent and teacher report of pragmatic aspects of communication: use of the children's communication checklist in a clinical setting. Dev. Med. Child Neurol. 43, 809-818. doi:10.1017/S0012162201001475

Brookman-Frazee, L., Stahmer, A., Baker-Ericzén, M. J., and Tsai, K. (2006). Parenting interventions for children with autism spectrum and disruptive behavior 
disorders: opportunities for cross-fertilization. Clin. Child Fam. Psychol. Rev. 9, 181-200. doi:10.1007/s10567-006-0010-4

Buitelaar, J. K., van der Wees, M., Swaab-Barneveld, H., and van der Gaag, R. J. (1999). Verbal memory and performance IQ predict theory of mind and emotion recognition ability in children with autistic spectrum disorders and in psychiatric control children. J. Child Psychol. Psychiatry 40, 869-881. doi:10.1111/1469-7610.00505

Canitano, R., and Scandurra, V. (2011). Psychopharmacology in autism: an update. Prog. Neuropsychopharmacol. Biol. Psychiatry 35, 18-28. doi:10.1016/j.pnpbp. 2010.10.015

Cantwell, D. P. (1996). Attention deficit disorder: a review of the past 10 years. J. Am. Acad. Child Adolesc. Psychiatry 35, 978-987. doi:10.1097/00004583-19960800000008

Carlson, C. L., and Mann, M. (2000). Attention-deficit/hyperactivity disorder, predominantly inattentive subtype. Child Adolesc. Psychiatr. Clin. N. Am. 9, 499-510, vi.

Carlsson, L. H., Norrelgen, F., Kjellmer, L., Westerlund, J., Gillberg, C., and Fernell, E. (2013). Coexisting disorders and problems in preschool children with autism spectrum disorders. ScientificWorldJournal 2013, 213979. doi:10.1155/ 2013/213979

Cooper, M., Martin, J., Langley, K., Hamshere, M., and Thapar, A. (2014). Autistic traits in children with ADHD index clinical and cognitive problems. Eur. Child Adolesc. Psychiatry 23, 23-34. doi:10.1007/s00787-013-0398-6

Cross-Disorder Group of the Psychiatric Genomics Consortium. (2013). Genetic relationship between five psychiatric disorders estimated from genome-wide SNPs. Nat. Genet. 45, 984-994. doi:10.1038/ng.2711

Davis, N. O., and Kollins, S. H. (2012). Treatment for co-occurring attention deficit/hyperactivity disorder and autism spectrum disorder. Neurotherapeutics 9, 518-530. doi:10.1007/s13311-012-0126-9

Di Martino, A., Zuo, X. N., Kelly, C., Grzadzinski, R., Mennes, M., Schvarcz, A., et al. (2013). Shared and distinct intrinsic functional network centrality in autism and attention-deficit/hyperactivity disorder. Biol. Psychiatry 74, 623-632. doi:10.1016/j.biopsych.2013.02.011

Eiraldi, R. B., Power, T. J., Karustis, J. L., and Goldstein, S. G. (2000). Assessing ADHD and comorbid disorders in children: the Child Behavior Checklist and the Devereux Scales of Mental Disorders. J. Clin. Child Psychol. 29, 3-16. doi:10.1207/S15374424jccp2901_2

Flicek, M. (1992). Social status of boys with both academic problems and attentiondeficit hyperactivity disorder. J. Abnorm. Child Psychol. 20, 353-366. doi:10.1007/ BF00918981

Frazier, T. W., Shattuck, P. T., Narendorf, S. C., Cooper, B. P., Wagner, M., and Spitznagel, E. L. (2011). Prevalence and correlates of psychotropic medication use in adolescents with an autism spectrum disorder with and without caregiverreported attention-deficit/hyperactivity disorder. J. Child Adolesc. Psychopharmacol. 21, 571-579. doi:10.1089/cap.2011.0057

Friedman, S. R., Rapport, L. J., Lumley, M., Tzelepis, A., VanVoorhis, A., Stettner, L., et al. (2003). Aspects of social and emotional competence in adult attentiondeficit/hyperactivity disorder. Neuropsychology 17, 50-58. doi:10.1037/08944105.17.1.50

Gadow, K. D., DeVincent, C. J., and Pomeroy, J. (2006). ADHD symptom subtypes in children with pervasive developmental disorder. J. Autism Dev. Disord. 36, 271-283. doi:10.1007/s10803-005-0060-3

Gadow, K. D., DeVincent, C. J., Pomeroy, J., and Azizian, A. (2004). Psychiatric symptoms in preschool children with PDD and clinic and comparison samples. J. Autism Dev. Disord. 34, 379-393. doi:10.1023/B:JADD.0000037415.21458.93

Gargaro, B. A., Rinehart, N. J., Bradshaw, J. L., Tonge, B. J., and Sheppard, D. M. (2011). Autism and ADHD: how far have we come in the comorbidity debate? Neurosci. Biobehav. Rev. 35, 1081-1098. doi:10.1016/j.neubiorev.2010.11.002

Geurts, H. M., Ridderinkhof, K. R., and Scholte, H. S. (2013). The relationship between grey-matter and ASD and ADHD traits in typical adults. J. Autism Dev. Disord. 43, 1630-1641. doi:10.1007/s10803-012-1708-4

Gillberg, C. (2010). The ESSENCE in child psychiatry: early symptomatic syndromes eliciting neurodevelopmental clinical examinations. Res. Dev. Disabil. 31, 1543-1551. doi:10.1016/j.ridd.2010.06.002

Greene, R. W., Biederman, J., Faraone, S. V., Ouellette, C. A., Penn, C., and Griffin, S. M. (1996). Toward a new psychometric definition of social disability in children with attention-deficit hyperactivity disorder. J. Am. Acad. Child Adolesc. Psychiatry 35, 571-578. doi:10.1097/00004583-199605000-00011
Grzadzinski, R., Di Martini, A., Brady, E., Mairena, M. A., O’Neale, M., Petrova, E., et al. (2011). Examining autistic traits in children with ADHD: does the autism spectrum extend to ADHD? J. Autism Dev. Disord. 41, 1178-1191. doi:10.1007/s10803-010-1135-3

Guttmann-Steinmetz, S., Gadow, K. D., and Devincent, C. J. (2009). Oppositional defiant and conduct disorder behaviors in boys with autism spectrum disorder with and without attention-deficit hyperactivity disorder versus several comparison samples. J. Autism Dev. Disord. 39, 976-985. doi:10.1007/s10803-009-0706-7

Hodgens, J. B., Cole, J., and Boldizar, J. (2000). Peer-based differences among boys with ADHD. J. Clin. Child Psychol. 29, 443-452. doi:10.1207/ S15374424JCCP2903_15

Holtmann, M., Bölte, S., and Poustka, F. (2007). Autism spectrum disorders: sex differences in autistic behaviour domains and coexisting psychopathology. Dev. Med. Child Neurol. 49, 361-366. doi:10.1111/j.1469-8749.2007.00361.x

Hosenbocus, S., and Chahal, R. (2013). Amantadine: a review of use in child and adolescent psychiatry. J. Can. Acad. Child Adolesc. Psychiatry 22, 55-60.

Hoza, B., Mrug, S., Gerdes, A. C., Hinshaw, S. P., Bukowski, W. M., Gold, J. A., et al. (2005). What aspects of peer relationships are impaired in children with attention-deficit/hyperactivity disorder? J. Consult. Clin. Psychol. 73, 411-423. doi:10.1037/0022-006X.73.3.411

Jahromi, L. B., Kasari, C. L., McCracken, J. T., Lee, L. S., Aman, M. G., McDougle, C. J., et al. (2009). Positive effects of methylphenidate on social communication and self-regulation in children with pervasive developmental disorders and hyperactivity. J. Autism Dev. Disord. 39, 395-404. doi:10.1007/s10803-008-0636-9

Klassen, A. F., Miller, A., and Fine, S. (2004). Health-related quality of life in children and adolescents who have a diagnosis of attention-deficit/hyperactivity disorder. Pediatrics 114, e541-e547. doi:10.1542/peds.2004-0844

Kotte, A., Joshi, G., Fried, R., Uchida, M., Spencer, A., Woodworth, K. Y., et al. (2013). Autistic traits in children with and without ADHD. Pediatrics 132, e612-e622. doi:10.1542/peds.2012-3947

Lecavalier, L., Gadow, K. D., DeVincent, C. J., and Edwards, M. C. (2009). Validation of DSM-IV model of psychiatric syndromes in children with autism spectrum disorders. J. Autism Dev. Disord. 39, 278-289. doi:10.1007/s10803-008-0622-2

Lee, D. O., and Ousley, O. Y. (2006). Attention-deficit hyperactivity disorder symptoms in a clinic sample of children and adolescents with pervasive developmental disorders. J. Child Adolesc. Psychopharmacol. 16, 737-746. doi:10.1089/cap.2006. 16.737

Leyfer, O. T., Folstein, S. E., Bacalman, S., Davis, N. O., Dinh, E., Morgan, J., et al. (2006). Comorbid psychiatric disorders in children with autism: interview development and rates of disorders. J. Autism Dev. Disord. 36, 849-861. doi:10.1007/s10803-006-0123-0

Marton, I., Wiener, J., Rogers, M., Moore, C., and Tannock, R. (2009). Empathy and social perspective taking in children with attention-deficit/hyperactivity disorder. J. Abnorm. Child Psychol. 37, 107-118. doi:10.1007/s10802-008-9262-4

Matson, J. L., Sipes, M., Fodstad, J. C., and Fitzgerald, M. E. (2011). Issues in the management of challenging behaviours of adults with autism spectrum disorder. CNS Drugs 25, 597-606. doi:10.2165/11591700-000000000-00000

Mikami, A. Y. (2010). The importance of friendship for youth with attentiondeficit/hyperactivity disorder. Clin. Child Fam. Psychol. Rev. 13, 181-198. doi:10.1007/s10567-010-0067-y

Milich, R., Landau, S., Kilby, G., and Whitten, P. (1982). Preschool peer perceptions of the behavior of hyperactive and aggressive children. J. Abnorm. Child Psychol. 10, 497-510. doi:10.1007/BF00920750

MTA Cooperative Group. (2004). National Institute of Mental Health Multimodal Treatment Study of ADHD follow-up: 24-month outcomes of treatment strategies for attention-deficit/hyperactivity disorder. Pediatrics 113, 754-761. doi:10.1542/peds.113.4.754

Mulligan, A., Anney, R. J., O’Regan, M., Chen, W., Butler, L., Fitzgerald, M., et al. (2009). Autism symptoms in attention-deficit/hyperactivity disorder: a familial trait which correlates with conduct, oppositional defiant, language and motor disorders. J. Autism Dev. Disord. 39, 197-209. doi:10.1007/s10803-0080621-3

Murray, M. J. (2010). Attention-deficit/hyperactivity disorder in the context of Autism spectrum disorders. Curr. Psychiatry Rep. 12, 382-388. doi:10.1007/ s11920-010-0145-3

Nijmeijer, J. S., Minderaa, R. B., Buitelaar, J. K., Mulligan, A., Hartman, C. A., and Hoekstra, P. J. (2008). Attention-deficit/hyperactivity disorder and social dysfunctioning. Clin. Psychol. Rev. 28, 692-708. doi:10.1016/j.cpr.2007.10.003 
Owen, R., Sikich, L., Marcus, R. N., Corey-Lisle, P., Manos, G., McQuade, R. D., et al. (2009). Aripiprazole in the treatment of irritability in children and adolescents with autistic disorder. Pediatrics 124, 1533-1540. doi:10.1542/peds.2008-3782

Pelham, W. E., Bender, M. E., Caddell, J., Booth, S., and Moorer, S. H. (1985). Methylphenidate and children with attention deficit disorder. Dose effects on classroom academic and social behavior. Arch. Gen. Psychiatry 42, 948-952. doi:10.1001/archpsyc.1985.01790330028003

Piven, J., Arndt, S., Bailey, J., and Andreasen, N. (1996). Regional brain enlargement in autism: a magnetic resonance imaging study. J. Am. Acad. Child Adolesc Psychiatry 35, 530-536. doi:10.1097/00004583-199604000-00020

Posey, D. J., Puntney, J. I., Sasher, T. M., Kem, D. L., and McDougle, C. J. (2004). Guanfacine treatment of hyperactivity and inattention in pervasive developmental disorders: a retrospective analysis of 80 cases. J. Child Adolesc. Psychopharmacol. 14, 233-241. doi:10.1089/1044546041649084

Posey, D. J., Wiegand, R. E., Wilkerson, J., Maynard, M., Stigler, K. A., and McDougle, C. J. (2006). Open-label atomoxetine for attention-deficit/hyperactivity disorder symptoms associated with high-functioning pervasive developmental disorders. J. Child Adolesc. Psychopharmacol. 16, 599-610. doi:10.1089/cap.2006.16.599

Rao, P. A., and Landa, R. J. (2013). Association between severity of behavioral phenotype and comorbid attention deficithyperactivity disorder symptoms in children with autism spectrum disorders. Autism 18:272-280. doi:10.1177/ 1362361312470494

Reiersen, A. M., Constantino, J. N., Volk, H. E., and Todd, R. D. (2007). Autistic traits in a population-based ADHD twin sample. J. Child Psychol. Psychiatry 48, 464-472. doi:10.1111/j.1469-7610.2006.01720.x

Riddle, M. A., Yershova, K., Lazzaretto, D., Paykina, N., Yenokyan, G., Greenhill, L., et al. (2013). Preschool attention deficit/hyperactivity disorder treatment study (PATS) 6-year follow-up. J. Am. Acad. Child Adolesc. Psychiatry 52, 264-278. doi:10.1016/j.jaac.2012.12.007

Rommelse, N. N., Franke, B., Geurts, H. M., Hartman, C. A., and Buitelaar, J. K. (2010). Shared heritability of attention-deficit/hyperactivity disorder and autism spectrum disorder. Eur. Child Adolesc. Psychiatry 19, 281-295. doi:10. 1007/s00787-010-0092-x

Rommelse, N. N., Geurts, H. M., Franke, B., Buitelaar, J. K., and Hartman, C. A. (2011). A review on cognitive and brain endophenotypes that may be common in autism spectrum disorder and attention-deficit/hyperactivity disorder and facilitate the search for pleiotropic genes. Neurosci. Biobehav. Rev. 35, 1363-1396. doi:10.1016/j.neubiorev.2011.02.015

Ronald, A., Edelson, L. R., Asherson, P., and Saudino, K. J. (2010). Exploring the relationship between autistic-like traits and ADHD behaviors in early childhood: findings from a community twin study of 2-year olds. J. Abnorm. Child Psychol. 38, 185-196. doi:10.1007/s10802-009-9366-5

Rossignol, D. A. (2009). Novel and emerging treatments for autism spectrum disorders: a systematic review. Ann. Clin. Psychiatry 21, 213-236.

Santosh, P. J., Baird, G., Pityaratstian, N., Tavare, E., and Gringras, P. (2006). Impact of comorbid autism spectrum disorders on stimulant response in children with attention deficit hyperactivity disorder: a retrospective and prospective effectiveness study. Child Care Health Dev. 32, 575-583. doi:10.1111/j.1365-2214.2006. 00631.x

Santosh, P. J., and Mijovic, A. (2004). Social impairment in hyperkinetic disorder relationship to psychopathology and environmental stressors. Eur. Child Adolesc. Psychiatry 13, 141-150. doi:10.1007/s00787-004-0372-4

Sharma, A., and Shaw, S. R. (2012). Efficacy of risperidone in managing maladaptive behaviors for children with autistic spectrum disorder: a meta-analysis. J. Pediatr. Health Care 26, 291-299. doi:10.1016/j.pedhc.2011.02.008

Sikora, D. M., Vora, P., Coury, D. L., and Rosenberg, D. (2012). Attention deficit/hyperactivity disorder symptoms, adaptive functioning, and quality of life in children with autism spectrum disorder. Pediatrics 130(Suppl. 2), S91-S97. doi:10.1542/peds.2012-0900G

Sinzig, J., Morsch, D., and Lehmkuhl, G. (2008). Do hyperactivity, impulsivity inattention have an impact on the ability of facial affect recognition in children with autism and ADHD? Eur. Child Adolesc. Psychiatry 17, 63-72. doi:10.1007/s00787-007-0637-9

St Pourcain, B., Mandy, W. P., Heron, J., Golding, J., Davey Smith, G., and Skuse, D. H. (2011). Links between co-occurring social-communication and hyperactiveinattentive trait trajectories. J. Am. Acad. Child Adolesc. Psychiatry 50, 892-902. doi:10.1016/j.jaac.2011.05.015

Subcommittee on Attention Deficit/Hyperactivity Disorder, Steering Committee on Quality Improvement and Management, Wolraich, M., Brown, L., Brown, R. T., DuPaul, G., et al. (2011). ADHD: clinical practice guideline for the diagnosis, evaluation, and treatment of attention-deficit/hyperactivity disorder in children and adolescents. Pediatrics 128, 1007-1022. doi:10.1542/peds.2011-2654

Uekermann, J., Kraemer, M., Abdel-Hamid, M., Schimmelmann, B. G., Hebebrand, J., Daum, I., et al. (2010). Social cognition in attention-deficit hyperactivity disorder (ADHD). Neurosci. Biobehav. Rev. 34, 734-743. doi:10.1016/j.neubiorev. 2009.10.009

van Steijn, D. J., Richards, J. S., Oerlemans, A. M., de Ruiter, S. W., van Aken, M. A., Franke, B., et al. (2012). The co-occurrence of autism spectrum disorder and attention-deficit/hyperactivity disorder symptoms in parents of children with ASD or ASD with ADHD. J. Child Psychol. Psychiatry 53, 954-963. doi:10.1111/j.1469-7610.2012.02556.x

Vora, P., and Sikora, D. (2011). Society for Developmental and Behavioral. San Antonio, TX: Pediatrics.

Whalen, C. K., Henker, B., and Hinshaw, S. P. (1985). Cognitive-behavioral therapies for hyperactive children: premises, problems, and prospects. J. Abnorm. Child Psychol. 13, 391-409. doi:10.1007/BF00912724

Williams, K., Brignell, A., Randall, M., Silove, N., and Hazell, P. (2013). Selective serotonin reuptake inhibitors (SSRIs) for autism spectrum disorders (ASD). Cochrane Database Syst. Rev. 8, doi:10.1002/14651858.CD004677.pub3

Yerys, B. E., Wallace, G. L., Sokoloff, J. L., Shook, D. A., James, J. D., and Kenworthy, L. (2009). Attention deficit/hyperactivity disorder symptoms moderate cognition and behavior in children with autism spectrum disorders. Autism Res. 2, 322-333. doi:10.1002/aur.103

Young, S., Heptinstall, E., Sonuga-Barke, E. J., Chadwick, O., and Taylor, E. (2005). The adolescent outcome of hyperactive girls: self-report of psychosocial status. J. Child Psychol. Psychiatry 46, 255-262. doi:10.1111/j.1469-7610.2004. 00350.x

Conflict of Interest Statement: The author declares that the research was conducted in the absence of any commercial or financial relationships that could be construed as a potential conflict of interest.

Received: 13 October 2013; accepted: 10 April 2014; published online: 29 April 2014. Citation: Leitner Y (2014) The co-occurrence of autism and attention deficit hyperactivity disorder in children - what do we know? Front. Hum. Neurosci. 8:268. doi: 10.3389/fnhum.2014.00268

This article was submitted to the journal Frontiers in Human Neuroscience.

Copyright (C) 2014 Leitner. This is an open-access article distributed under the terms of the Creative Commons Attribution License (CC BY). The use, distribution or reproduction in other forums is permitted, provided the original author(s) or licensor are credited and that the original publication in this journal is cited, in accordance with accepted academic practice. No use, distribution or reproduction is permitted which does not comply with these terms. 